\title{
Diffraction Contrast Tomography in the Laboratory - Applications and Future Directions
}

\section{Holzner, ${ }^{1}$ L. Lavery, ${ }^{1 *}$ H. Bale, ${ }^{1}$ A. Merkle, ${ }^{1}$ S. McDonald, ${ }^{2}$ P. Withers,${ }^{2}$ Y. Zhang, ${ }^{3}$ \\ D. Juul Jensen, ${ }^{3}$ M. Kimura, ${ }^{4}$ A. Lyckegaard, ${ }^{5}$ P. Reischig, ${ }^{5}$ and E.M. Lauridsen ${ }^{5}$}

${ }^{1}$ Carl Zeiss X-ray Microscopy Inc., 4385 Hopyard Rd., Pleasanton, CA 94588

${ }^{2}$ Manchester X-ray Imaging Facility, School of Materials, University of Manchester, Manchester, M13 9PL, UK

${ }^{3}$ Department of Wind Energy, Technical University of Denmark, Fredriksborgvej 399, 4000 Roskilde, Denmark

${ }^{4}$ Institute of Materials Structure Science (IMSS), High Energy Accelerator Research Organization (KEK), 1-1 Oho,

Tsukuba, Ibaraki, 305-0801, Japan

${ }^{5}$ Xnovo Technology ApS, Galoche Alle 15, 4600 Køge, Denmark

*leah.lavery@zeiss.com

Abstract: LabDCT derives 3D crystallographic information via diffraction contrast tomography (DCT) within a commercial laboratory X-ray microscope (ZEISS Xradia 520 Versa) that uses a synchrotron-style detection system for tomography. The establishment of DCT into a laboratory setting opens the way for routine, non-destructive, time-evolution studies of grain structure over meaningful sample volumes. The combination of grain information with microstructural features such as cracks, porosity, and inclusions, all derived non-destructively in $3 \mathrm{D}$, enables materials characterization of damage, deformation, and growth mechanisms. Here, we introduce LabDCT and demonstrate its capabilities through a selection of materials science applications.

\section{Introduction}

Determining 3D crystallographic information holds tremendous value for $3 \mathrm{D}$ materials science because the properties and performance of materials are intricately linked to microstructural morphology. Conventional 2D approaches (for example, metallography) have been extended to 3D through serial methods but still require destructive sectioning of sample material. Achieving direct non-destructive visualization of 3D crystallographic structure was first possible by diffraction contrast tomography (DCT) in the early 2000s at synchrotron X-ray facilities; it is today only available at a limited number of sites around the world. Recent developments, however, have made DCT possible on a laboratory X-ray microscope.

The first laboratory-based DCT system (LabDCT ${ }^{\mathrm{TM}}$ from Carl Zeiss $\mathrm{X}$-ray Microscopy) for 3D grain imaging is now available and includes advanced reconstruction and analysis capabilities [1]. The establishment of

(c) (a)

DCT into a laboratory setting opens the way for routine, non-destructive, time-evolution studies of grain structure over meaningful sample volumes up to $8 \mathrm{~mm}^{3}$. This extends DCT access beyond the synchrotron and complements electron

(b)
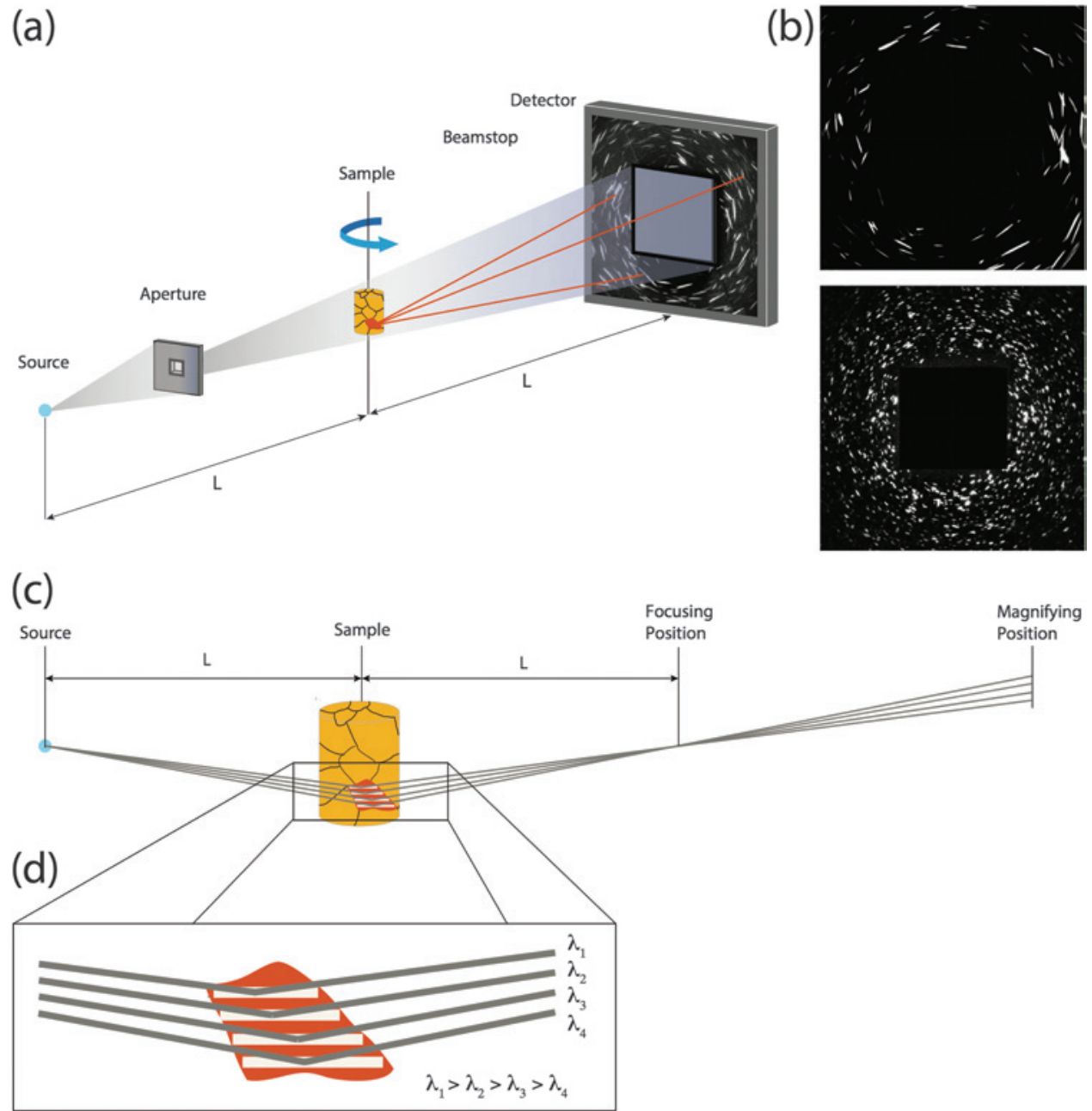

Figure 1: (a) Schematic of the LabDCT implementation on the ZEISS Xradia 520 Versa X-ray microscope. (b) LabDCT diffraction patterns from two different Ti-alloy samples: (top) a sample with a few relatively large grains has a pattern with few large lines, and (bottom) a sample with many small grains has a pattern with short lines closely spaced. (c) Laue-focusing effect in which a crystal grain acts as a cylindrical lens focusing polychromatic and divergent $X$-ray beam into a line instead of spots in the diffraction pattern. (d) Enlarged area from (c) showing the energy gradient across the diffracting grain. 


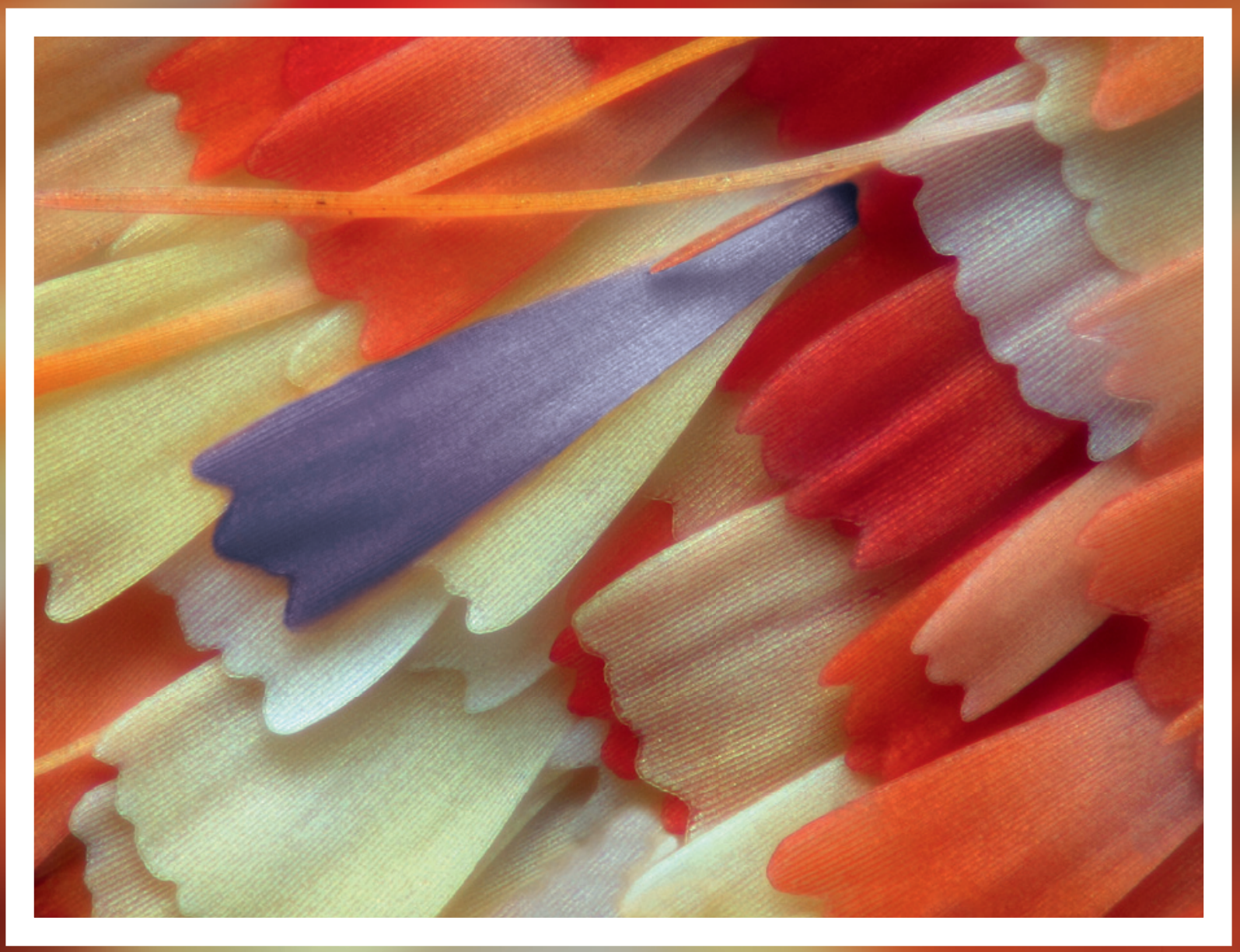

\section{BIG DISCOVERIES IN SMALL DETAILS. WE GET THE PICTURE.}

\section{THE BASLER POWERPACK FOR MICROSCOPY DELIVERS EVERYTHING YOU NEED FOR DYNAMIC MICROSCOPIC IMAGING IN A BUNDLED SOLUTION TAILORED TO YOUR EXACT SPECIFICATIONS.}

Basler has been developing superb imaging technology for more than 25 years. Now, we're using our history of innovation to drive future trends of microscopy. Introducing the Basler PowerPack for Microscopy - an accurate, affordable bundle of microscopy components that deliver superior images, premier performance and precise results. It can be utilized for applications such as education, biomedical or life science, as well as diagnostics. Basler is giving you yet another premium product at an exceptional value.

Think you know the object in focus? Test yourself at baslerweb.com/MPP.

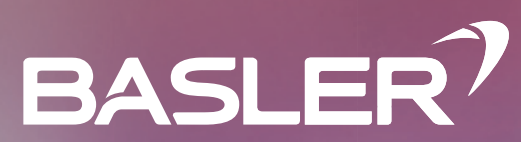

the power of sight

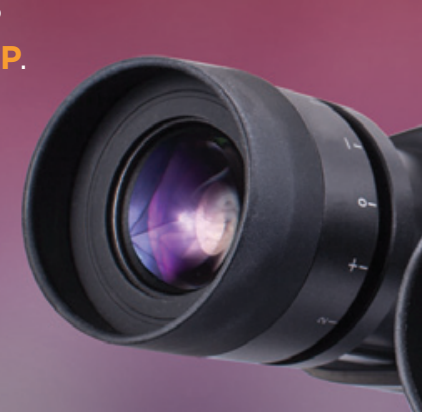


(a)

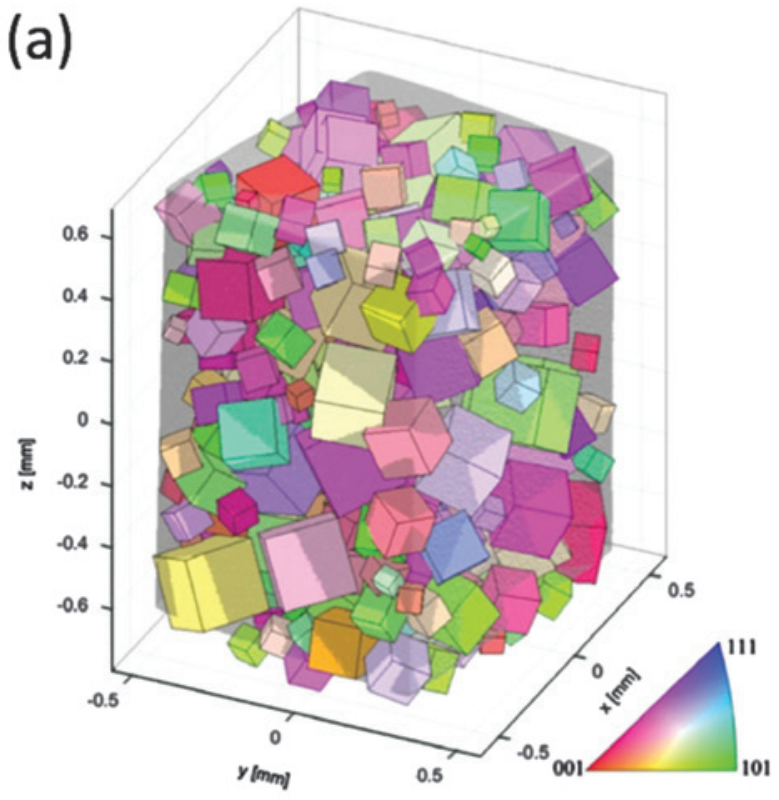

(b)

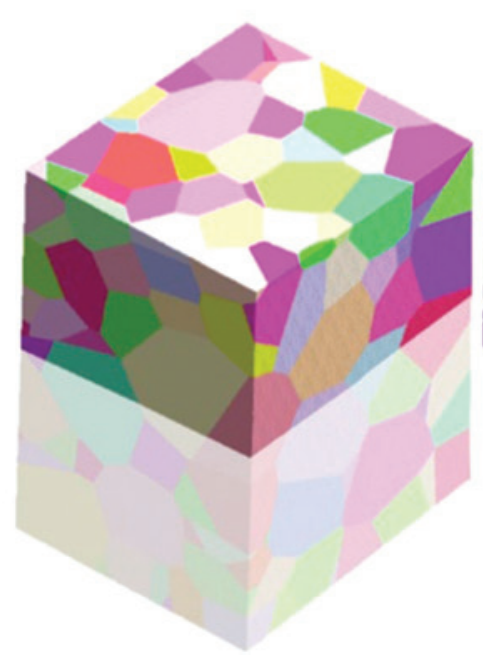

LabDCT

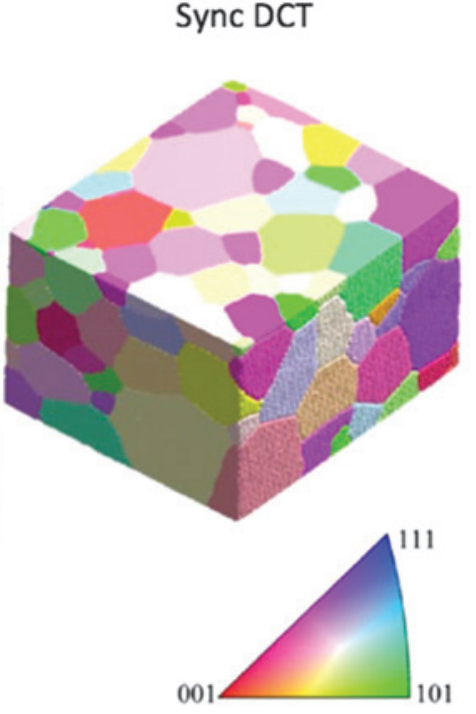

(c)

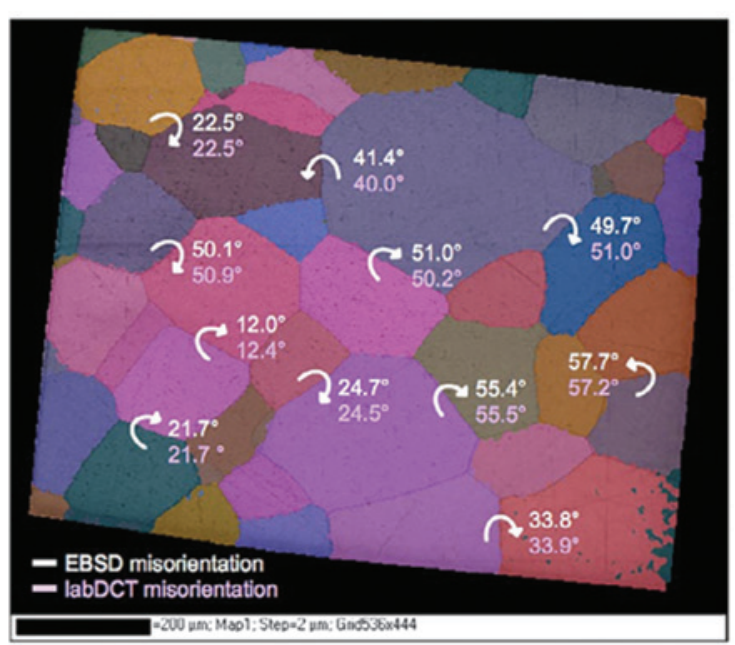

(d)

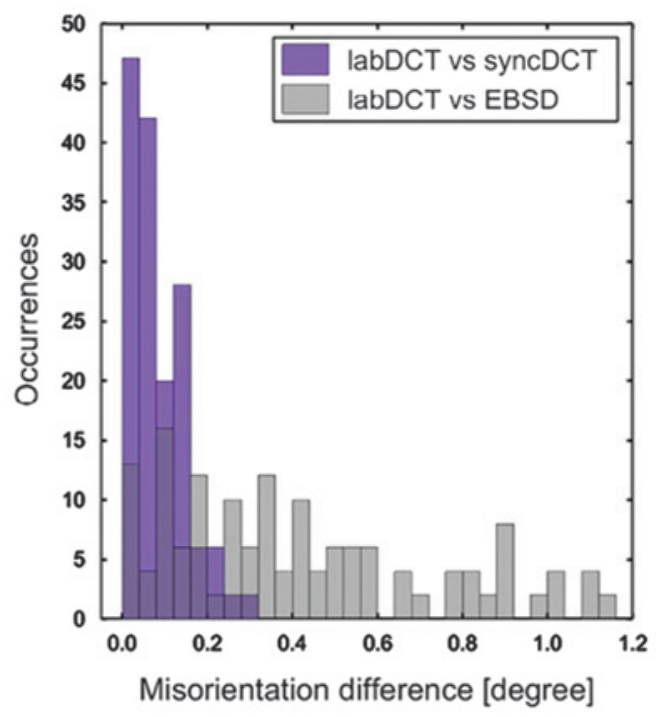

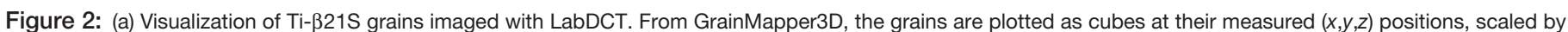

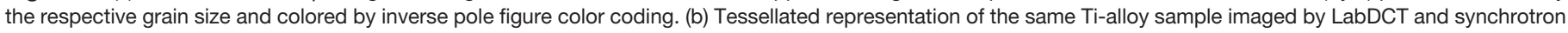

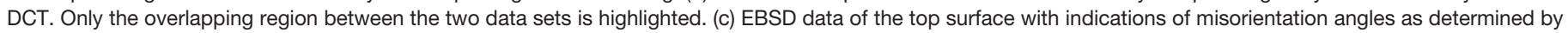
EBSD and LabDCT. (d) Histogram of the differences in misorientation measured with LabDCT/synchrotron DCT and LabDCT/EBSD.

backscatter diffraction (EBSD) end-point characterization and other crystallographic imaging techniques at finer scales such as TEM-based orientation imaging microscopy. The combination of grain information with microstructural features such as cracks, porosity, and inclusions, all derived non-destructively in $3 \mathrm{D}$, enables materials characterization of damage, deformation, and growth mechanisms. In this article we introduce the LabDCT technique and demonstrate its capabilities through a selection of application examples in materials science. We also discuss innovative methods to extend the current capabilities of the technology.

\section{Road to LabDCT}

Since its introduction in the 1980s, the EBSD technique has provided detailed 2D surface grain orientation maps in scanning electron microscopes [2-3]. Samples require mechanical (and sometimes ion) polishing to assure a damage-free surface. The EBSD method can be extended to 3D by serial sectioning, typically using a focused ion beam (FIB) to mill sections. Higher spatial resolution is achieved, albeit for relatively small volumes [4]. Because of the destructive nature of data acquisition, 3D EBSD cannot be used for in-situ or $4 \mathrm{D}$ experiments.

Mapping crystallography in 3D is available at synchrotron light sources using extremely high-flux monochromatic X-ray beams through techniques such as synchrotron DCT [5-6] and 3D X-ray diffraction microscopy (3DXRD) [7-9]. Synchrotron DCT provides crystallographic information and the sample's microstructure in $3 \mathrm{D}$. It has enabled microstructure investigations linked to 

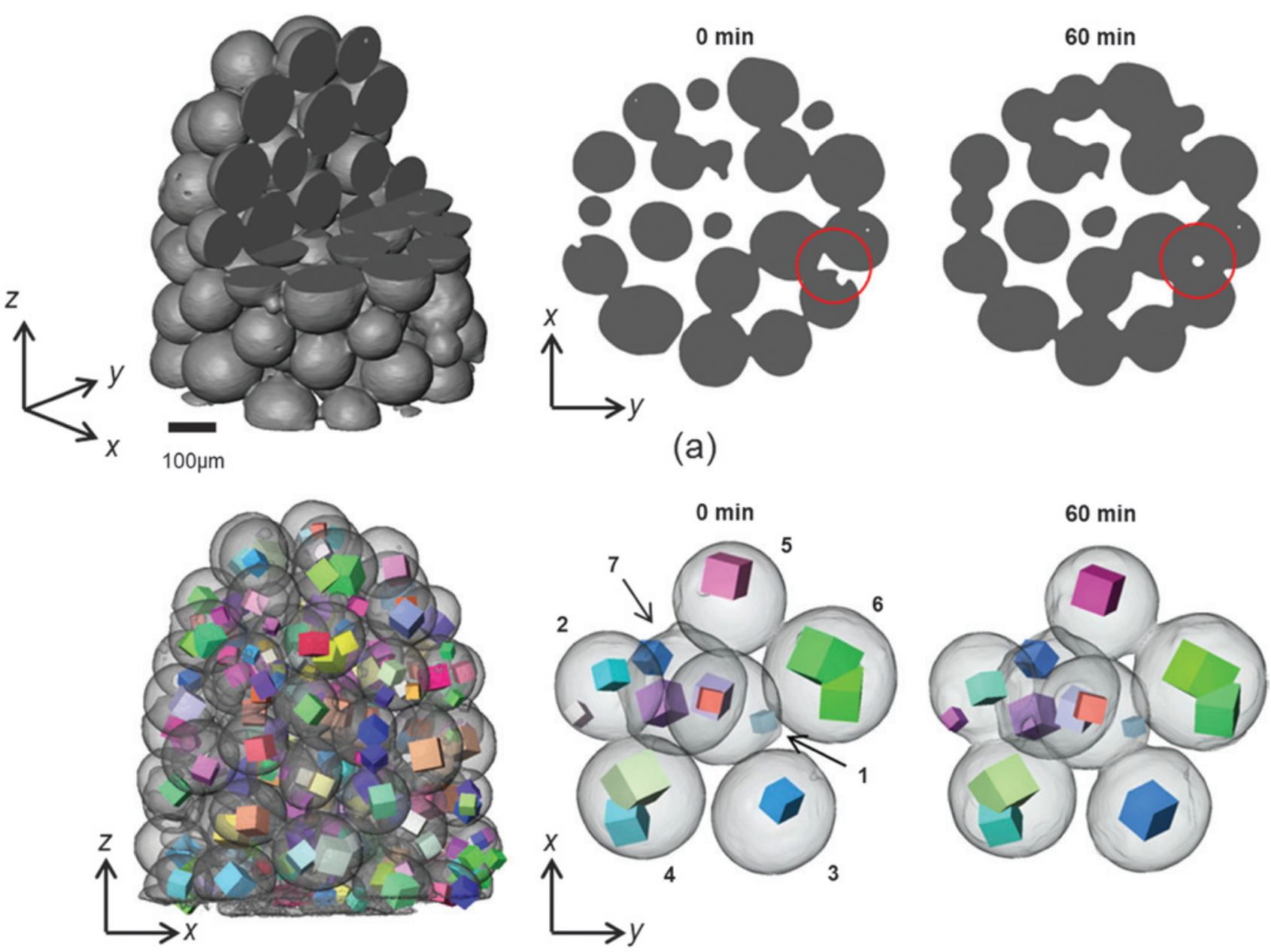

(b)

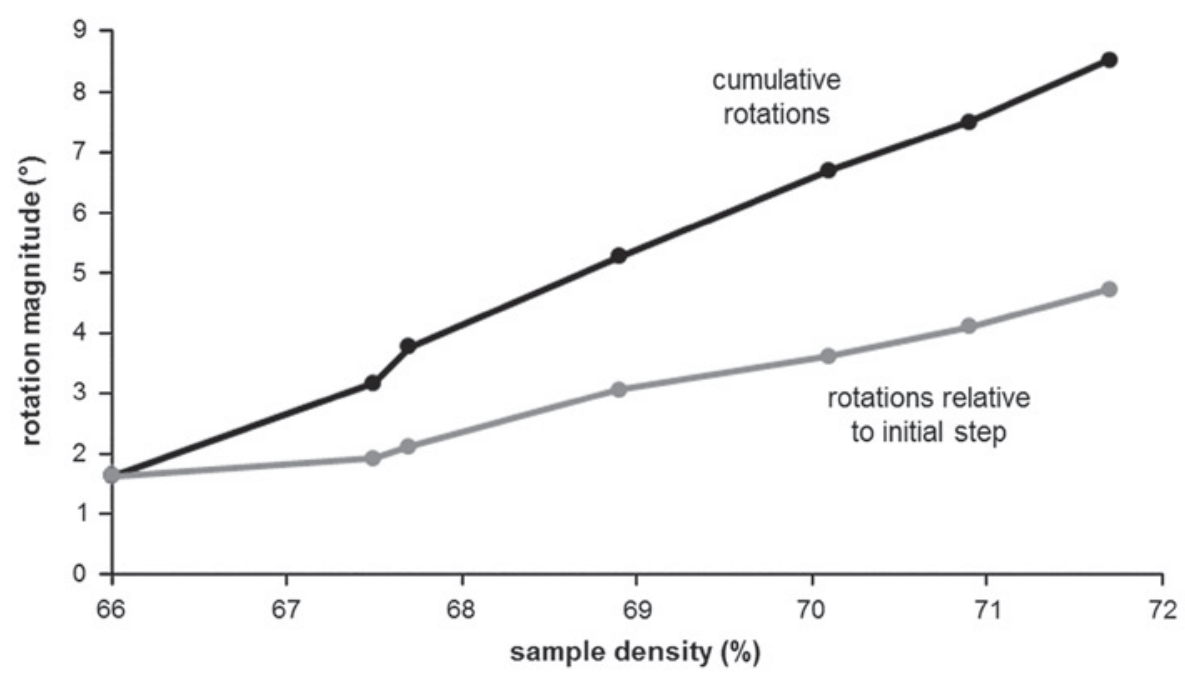

(c)

Figure 3: Sintering of Cu particle powder. (a) Tomographic rendering of powder at $t=0$ min, as well as virtual slices through the volume from the initial state and after a sintering time of $60 \mathrm{~min}$ at $1050^{\circ} \mathrm{C}$. (b) LabDCT analysis revealing the crystallographic information of the entire sample. Sub-region showing a detailed view for comparison of the grain orientations of seven neighboring $\mathrm{Cu}$ spheres. (c) Each grain within a single particle can provide an independent observation of the particle rotation. The average cumulative rotation of all particles was calculated to a total angular rotation of $8.5^{\circ}$.

stress corrosion cracking [10], fatigue [11-12], and temporal reorientation during sintering [13]. Yet only a few synchrotron facilities worldwide offer DCT, an indication of a need to develop the capability with wider availability and accessibility. 
(a)

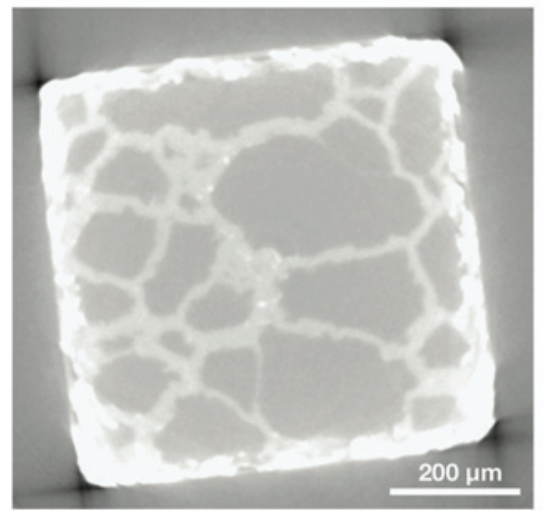

(b)

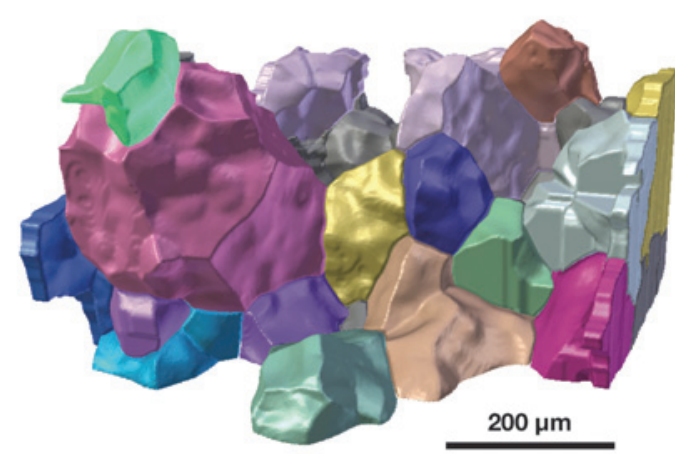

Figure 4: Grain morphology investigation on a commercial aluminum alloy. (a) Virtual slice through tomographic reconstruction volume of aluminum sample treated with gallium. The grain boundaries decorated with gallium are clearly visible against the darker aluminum grains. Using these decorations, the shape of all grains can be obtained in 3D. The combination of such information together with crystallography acquired by LabDCT allows visualization of the complete sample microstructure. (b) Rendering of the grain morphology combined with grain orientation information (color) from LabDCT.

LabDCT is the first DCT system for 3D grain mapping that uses a laboratory X-ray microscope (ZEISS Xradia 520 Versa). X-ray microscopes (XRM) incorporate a number of $\mathrm{X}$-ray optical elements that have driven resolution and contrast to levels previously unachievable by conventional computed tomography (CT) instrumentation [14]. ZEISS sub-micron 3D X-ray microscopes employ advanced detectors developed at synchrotron facilities and couple them to high-energy polychromatic laboratory X-ray sources. The post-sample dual-stage imaging detector uses both geometric and optical magnification and provides a small and tunable pixel size. The detector design expands source-sample working distance and improves the standard trade-off of sample size versus resolution found in conventional laboratory micro-CT. Furthermore, the XRM architecture enables propagation phase contrast for low-absorption contrast imaging, high-resolution analysis of larger samples, in addition to the $3 \mathrm{D}$ grain imaging modality, LabDCT.

\section{LabDCT: How it Works}

A schematic representation of the LabDCT implementation is shown in Figure 1. The X-ray beam is constrained through an aperture to illuminate the sample (Figure 1a). Diffraction information and the standard (absorption-contrast) CT information are collected sequentially and automatically in one batch of scans. A beam-stop after the sample blocks transmitted $\mathrm{X}$ rays to increase sensitivity to the diffraction signals. LabDCT measurements are followed by a computational reconstruction using GrainMapper3Dadvanced reconstruction and analysis software developed by Xnovo Technology [15]. During the acquisition stage, the first scan collects projections at increments through 360 degrees in the direct beam from which a 3D reconstruction of the absorption signal is created, as in traditional X-ray tomography. Subsequently, the source, sample, and detector are placed in a symmetric Laue geometry (Figures $1 \mathrm{a}$ and $1 \mathrm{c}$ ) in which diffraction images are collected. The diffraction patterns are acquired on the outer region of the detector (Figure 1b) for grain structure, are exported into an open data format suitable for additional subsequent analysis using custom analysis software and/or simulation tools.

LabDCT uses an X-ray beam that is divergent and polychromatic, which results in a Laue focusing effect. A crystal grain acts as a cylindrical lens focusing $\mathrm{X}$ rays into a line instead of spots in the diffraction pattern (Figure 1b). Within this grain, there is a set of crystal planes fulfilling the Bragg condition. Across the direction perpendicular to the crystal planes, the grain intercepts $\mathrm{X}$ rays over a span of incident angles from the source. The Bragg condition can be fulfilled across the whole angular span of the grain given the poly-chromaticity of the incoming X-ray beam, resulting in a linear energy gradient contributing to the diffraction across the grain as illustrated in Figures $1 \mathrm{c}$ and $1 \mathrm{~d}$. As a result, the incoming $\mathrm{X}$ rays are focused into a line because no Bragg diffraction occurs in the orthogonal direction.

Adapting synchrotron DCT and bringing it to a laboratory setting is a nontrivial task-especially because laboratory X-ray sources have orders of magnitude less flux, which would result in impractically long scan times. Compared to the parallel and monochromatic X-ray source typically used in the synchrotron DCT technique, the source for LabDCT is polychromatic and operates at higher energy (up to $160 \mathrm{kV}$ ). This gives rise to many more diffraction events for a single sample orientation, and thus the information content per projection image is increased. Therefore samples can be analyzed by collecting far fewer projections (up to a factor of 50 fewer) than in synchrotron DCT. The Laue focusing geometry improves diffraction signal detection and allows the handling of many closely spaced reflections; focusing the diffracted signal into a line increases signalto-noise ratio and therefore the sensitivity. Furthermore, for diffraction experiments using monochromatic $\mathrm{X}$ rays, one needs to rock the sample during data acquisition to integrate the diffraction signal. When using a polychromatic $\mathrm{X}$-ray beam, such rocking is not required, simplifying data 
acquisition. Uniquely, the higher X-ray energy of lab sources yields higher penetration depth allowing the DCT imaging of denser samples with wider cross sections compared to synchrotron DCT. LabDCT enables 3D crystallographic imaging over meaningful sample volumes spanning up to $8 \mathrm{~mm}^{3}$, roughly three orders of magnitude larger than typical 3D EBSD imaging volumes.

Currently, LabDCT determines the grain position, grain size, and grain orientation as shown in Figure 2a. In a typical visualization, the individual body-centered cubic grains in a $\beta$-titanium alloy (stable at room temperature) are represented by cubes indicating the orientation of the crystallographic unit cell. The color chosen reflects the crystallographic directions with respect to the $z$-axis, and the cube size is scaled according to grain size. A more accurate depiction of the grain morphology can be produced using a spacefilling representation of the grain microstructure computed by an advanced Laguerre-tessellation (Figure $2 \mathrm{~b}$ ) based on volume and centroid information [16]. These informationrich 3D datasets can be used for further analysis and representations in terms of the number of grain neighbors, neighboring crystal orientation, and grain position and volume. While both LabDCT and synchrotron DCT are non-destructive in nature, the LabDCT configuration, combining geometric magnification and Laue focusing, adds an additional advantage by facilitating more space around the sample allowing for the incorporation of sample environments like furnaces, load rigs, etc.
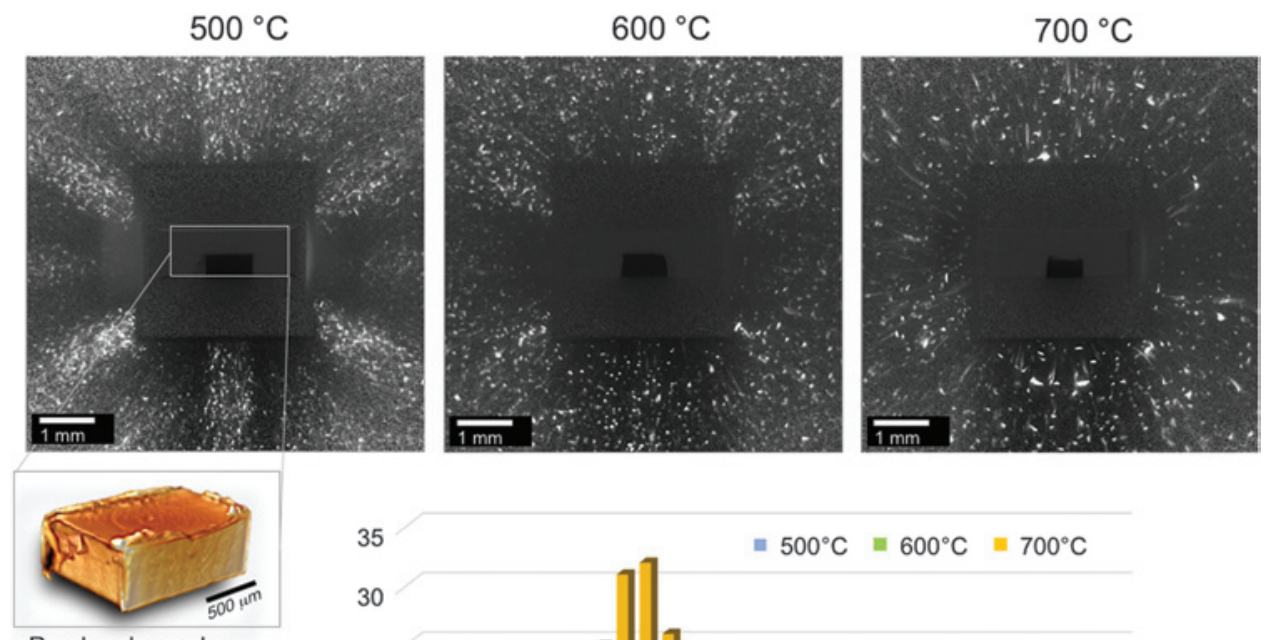

Rendered sample volume

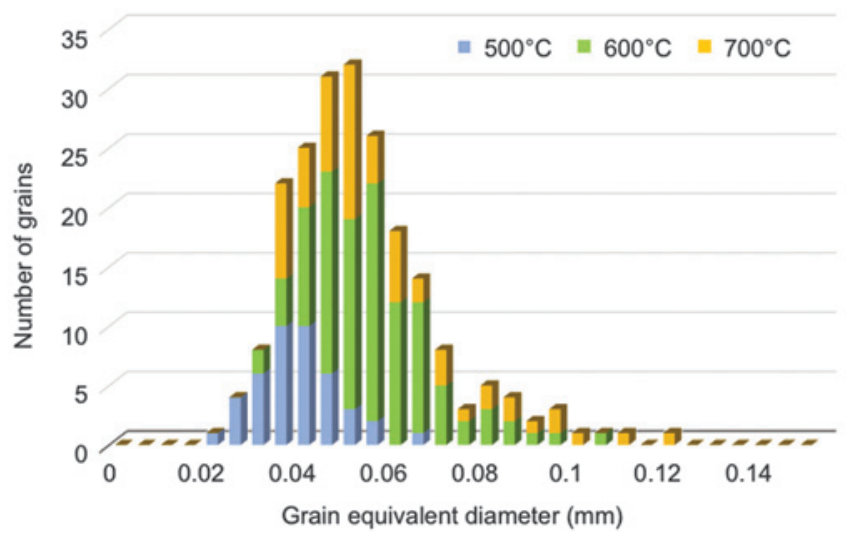

Figure 5: Recrystallization in production steel. LabDCT tracked grain recrystallization process as a function of grain size and annealing temperature. The top row shows example diffraction patterns collected at various temperatures. The graph at the bottom shows the corresponding distribution of grain sizes as determined from the LabDCT reconstruction.
The validity of LabDCT has been established through good agreement with experimental comparisons to EBSD and synchrotronDCT tomography;seeFigures $2 b-d$ and [1]. Figure $2 d$ shows a histogram of the differences in measured misorientation related to LabDCT compared with synchrotron DCT, and LabDCT compared with EBSD. The DCT techniquesboth being X-ray based-agree well within their accuracy, whereas the differences between LabDCT and EBSD approach 1 degree because of the lower angular resolution of the EBSD technique [17].

\section{LabDCT Materials Science Applications}

The properties of polycrystalline metallic and ceramic engineering materials of interest are affected by the underlying crystalline grain structure. Studying grain structure in 3D and with respect to process conditions aids in designing materials optimized for their intended application environment. The ability to track a material's response to applied mechanical load or temperature, for example, over time during repeated observations (for example, " $4 \mathrm{D}$ " studies) while being able to monitor not just the microstructure but also the underlying crystallographic information, would certainly be a remarkable capability. In the following, we highlight applications of LabDCT pertaining to different material systems.

Sintering of copper metal powder. Sintering of loose or compacted granular bodies has a strong influence on the final microstructure and properties of structural components produced via the powder metallurgical route [18]. The sintering process is accompanied by dimensional changes of the body such as particle translation, rotation, and changes to internal pore structure associated with the growth of necks between neighboring particles [19-20]. Long sintering times are needed to eliminate large pores via consolidation by interfacial and bulk diffusion. Unfortunately, this can lead to excessive grain growth, which can have a detrimental effect on the performance of the final component. For optimal performance, growth mechanisms must be understood and controlled to achieve full densification with limited grain growth.

A sample containing spherical polycrystalline copper particles was 3D-imaged before and after sintering at $1050^{\circ} \mathrm{C}$ for 60 minutes in an external tube furnace under an inert atmosphere. The reconstructed volume from the $\mathrm{Cu}$ powder sample is shown in Figure 3a. From both initial and post-sintered datasets, a virtually sectioned equivalent slice was selected from the tomography volume. The shape changes that occurred during the 


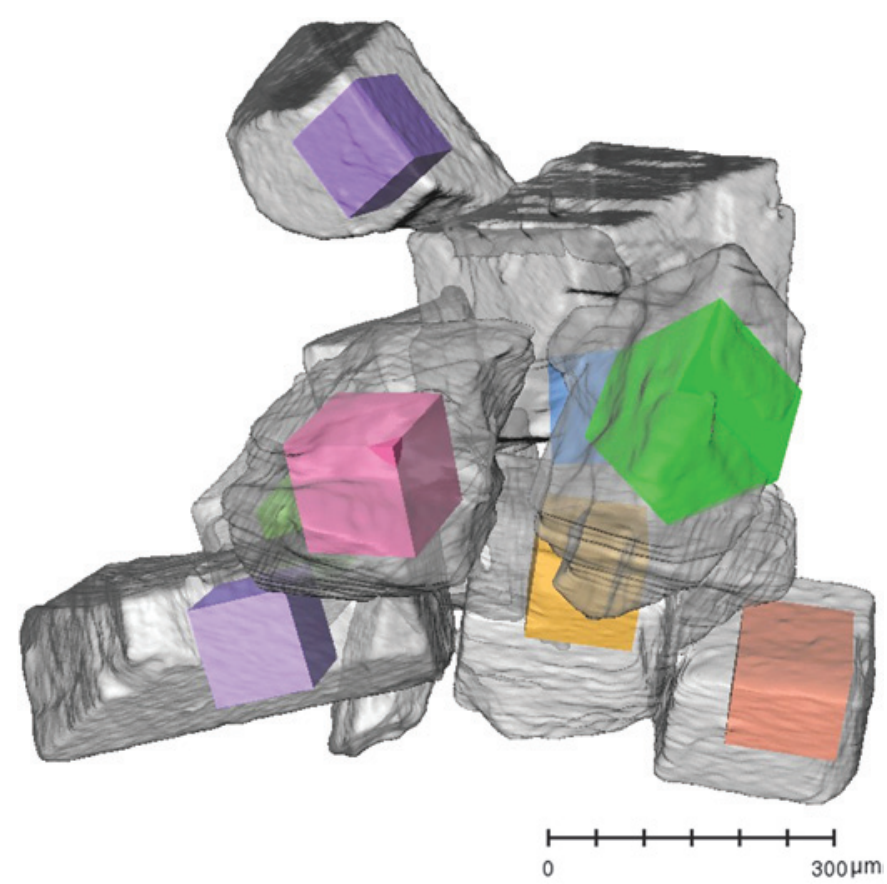

Figure 6: Rock salt embedded in a polymer matrix. The rendering represents the individual salt grains in light gray together with the cube reconstruction from LabDCT.

development and growth of inter-particle contacts, or "necks," between neighboring particles are highlighted. It was found that these shape changes are accompanied by a $\sim 5.7 \%$ increase in density over the timescale studied as determined by absorption tomography.

Figure $3 b$ shows a grain map reconstructed from LabDCT analysis of the copper particle sample. Each grain is represented by a cube showing its centroid position and orientation overlaid on the tomography image. Grain size is displayed in relative terms by the width of the cube representing the grain radius. In this case, the imaged volume contained 91 particles (mean particle size was $100 \mu \mathrm{m}$ ) with about 200 grains, giving an average of 2.2 grains per particle. For clear illustration, the grains within a small group of 7 neighboring particles located at the top of the sample have been extracted. Each individual grain can be tracked before and after sintering. Although the early stages of densification studied here did not show any significant growth of grains (Figure $3 \mathrm{~b}$ ), the shape changes occurring were primarily associated with particle rearrangements and rotations of the particles. From the reconstructed LabDCT grain maps, the magnitudes of rotation of the grains, and thus the particles, can be calculated. Each grain within a single particle can provide an independent observation of the particle rotation. The average cumulative rotation of all particles in the sample is shown in Figure $3 \mathrm{c}$ with a total angular rotation of $8.5^{\circ}$. It is interesting to note that the particles that rotate the least, particles 1 and 7 , are the particles with a larger number of inter-particle contacts. As the number of inter-particle contacts increases, the rotation of a particle becomes more restricted because the crystallographic misorientation between the particle and its neighbor provides the driving force for rotation. These experiments provide an improved theoretical description of the early stages of the sintering process.

Grain morphology in aluminum. 3D morphological and crystallographic information was determined for grains in an aluminium-gallium system. A commercial aluminum alloy (AA1050) was annealed at $600^{\circ} \mathrm{C}$ for 4 hours to provide an average grain size of 80 micrometers. To highlight the grain boundary structure in the aluminium alloy, liquid gallium $\left(\mathrm{T}_{\mathrm{m}}=302.9 \mathrm{~K}\right)$ was placed in direct contact with the aluminium surface at $100^{\circ} \mathrm{C}$ for two hours in order to penetrate and wet the grain boundaries. The Ga decorations enhance visualization of the grain boundary network in the absorption tomography image (Figure 4a). 3D grain representation based on tessellations can provide information on grain neighbor relationships. Despite their accuracy, tessellations remain an approximation and do not provide information such as grain boundary curvature. In the Al-Ga system, combining grain shapes, from the segmented absorption scan, with the LabDCT crystallographic information provides a high-fidelity description of the 3D microstructure (Figure $4 \mathrm{~b}$ ). From these data, important microstructural quantification such as full $5 \mathrm{D}$ grain boundary characterization can be derived.

Recrystallization in production steel. Well-controlled rolling of steels is needed to produce consistent microstructures to meet stringent material specifications such as strength and toughness. To produce such structures consistently requires a thorough understanding of the effects of compositional and process variables on grain refinement at every stage of the production process. The rolling process often introduces grain shape and orientation changes during the deformation, and grains may acquire a preferred orientation or texture relative to the direction of applied stress. An annealing step above the recrystallization temperature produces new strain-free grains. Following the changes in grain size, LabDCT tracked the grain recrystallization process as a function of temperature of annealing for a production steel (Figure 5). Further LabDCT analysis can characterize the $3 \mathrm{D}$ grain structure relative to the rolling direction.

Sodium chloride in 3D. The feasibility of LabDCT was recently explored for a non-metallic, low-density material, sodium chloride. In addition to its familiar domestic uses, sodium chloride is mined for various high-volume chemical industry applications. For LabDCT analysis, a granular $\mathrm{NaCl}$ sample was imaged within a polymer matrix. Figure 6 shows the identification of grain positions and orientations rendered together with the $3 \mathrm{D}$ image. The measured grain orientations of the cubes matched the cleaved (100) crystal facets providing encouraging results for extending the use of LabDCT to other low-density, non-metallic material systems.

\section{Discussion}

The results here illustrate the capability of linking crystallographic and grain microstructure information with that obtained via conventional X-ray microscopy in absorption or phase contrast imaging. That this can be accomplished 
non-destructively in $3 \mathrm{D}$ in a laboratory setting (for example, not only at a synchrotron) creating a powerful tool to access numerous opportunities in materials research. LabDCT is now routinely possible on an X-ray microscope with a polychromatic laboratory source, taking advantage of the Laue focusing effect. The technique has been successfully demonstrated for several materials science applications and will pave the way for routine, non-destructive studies of the time-evolution of grain structure that can complement destructive 3D EBSD end-point characterization and other crystallographic imaging techniques such as TEM-based orientation imaging microscopy. The results from LabDCT were compared against the well-established methods for determining grain orientation such as synchrotron-based DCT and EBSD. Samples with more grains and/or larger volumes can be imaged with fewer projections in LabDCT compared to synchrotron DCT, while achieving total scan times in a manageable range. Limitations for sample characteristics are very similar between the synchrotron and lab technique, where the best results for both are with samples that are undeformed with grains being equiaxed and unstrained. Direct morphology determination is possible in principle, but not in the scope of this introductory article and will be further developed in the near future. Other advanced analysis capabilities, such as in multi-phase materials or strain analysis, currently being performed at synchrotron DCT systems beam lines will eventually also be possible with LabDCT. First estimations show that strain sensitivity for LabDCT of up to $10^{-4}$ is achievable. Combining non-destructive $3 \mathrm{D}$ grain orientation information with microstructural features such as cracks, porosity, and inclusions opens new possibilities for characterizing damage, deformation, and growth phenomena in $3 \mathrm{D}$ materials science. Data acquired serve as valuable inputs to materials modeling platforms, especially for reducing the need for statistical sampling when performing materials evolution studies now that the same region of the same sample may be observed and quantified over time. 3D grain orientation data are also valuable inputs into multi-scale, multi-layered modeling platforms that can virtually evaluate mechanical properties to produce high-fidelity simulation results. This type of capability should open doors for $3 \mathrm{D}$ materials science for many years to come.

\section{Conclusion}

LabDCT has the ability to derive $3 \mathrm{D}$ crystallographic information via DCT within a commercial laboratory X-ray microscope (ZEISS Xradia 520 Versa) that uses a synchrotronstyle detection system for non-destructive tomography. LabDCT has been applied to several applications such as the production of steel, automotive alloys, and thermal sintering processes. Analysis is powered by GrainMapper3D, an advanced reconstruction and analysis software developed by Xnovo Technology, yet designed intuitively for the non-expert user.

\section{References}

[1] SA McDonald et al., Sci Rep 5 (2015) 14665.

[2] JA Venables and CJ Harland, Philos Mag 27 (1973) 1193-1200.

[3] DJ Dingley and DP Field, Mater Sci Technol 13 (1997) 69-78.

[4] DJ Rowenhorst et al., Acta Mater 58 (2010) 5511-19.

[5] W Ludwig et al., JOM 62 (2010) 22-28.

[6] P Reischig et al., J Appl Crystallogr 46 (2013) 297-311.

[7] EM Lauridsen et al., J Appl Cryst 34 (2001) 744-50.

[8] HF Poulsen, Three-Dimensional X-ray Diffraction Microscopy: Mapping Polycrystals and their Dynamics, Springer, Berlin, 2004.

[9] S Schmidt et al., Scr Mater 59 (2008) 491-94.

[10] A King et al., Science 321 (2008) 382-85.

[11] M Herbig et al., Acta Mater 59 (2011) 590-601.

[12] A King et al., J Acta Mater 59 (2011) 6761-71.

[13] SA McDonald et al., Proc. 33rd Risø Intl Symp Mat Sci, eds. S Faester et al., Risø, Denmark, Tech. Univ. Denmark, 2012, 93-105.

[14] A Merkle and J Gelb, Microscopy Today 21 (2013) 10-15.

[15] Xnovo Technology, http://xnovotech.com.

[16] A Lyckegaard et al., Advanced Engineering Materials 13(3) (2011) 165-70.

[17] D Stojakovic, Process Appl Ceramics 6(1) (2012) 1-13.

[18] ACF Cocks, Prog Mater Sci 46 (2001) 201-29.

[19] R Grupp et al., Nat Commun 2 (2011) 298-303.

[20] HE Exner and C Müller, J Am Ceram Soc 92 (2009) 1384-90.

\section{Precision, Speed, Stability \\ NANO-POSITIONING FOR MICROSCOPY}

\section{PI}

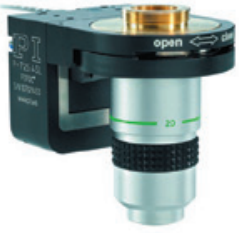

High-speed nanofocus $<1 \mathrm{~nm}$ resolution

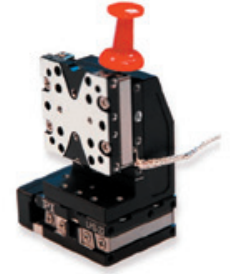

Miniature piezo motors

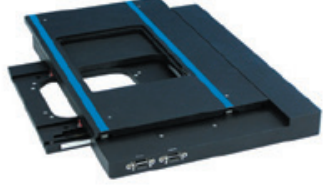

High-stability $X Y$ piezo motor stage, $100 \mathrm{~mm}$

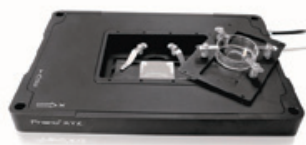

$X Y Z$ flexure stage $<1 \mathrm{~nm}$ resolution

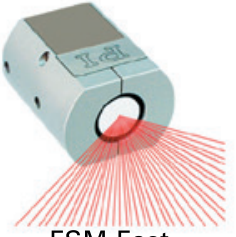

FSM Fast

Beam Steering

PI (Physik Instrumente) LP · Auburn, MA · info@pi-usa.us · 508-832-3456

PRECISION MOTION CONTROL \& POSITIONING SOLUTIONS 


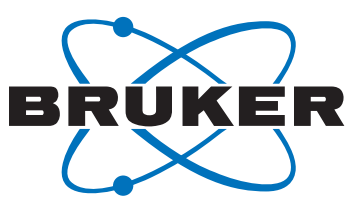

Someone has to be first.

\section{XFlash ${ }^{\circledR}$ FlatQUAD, multiple detector systems \& VZ}

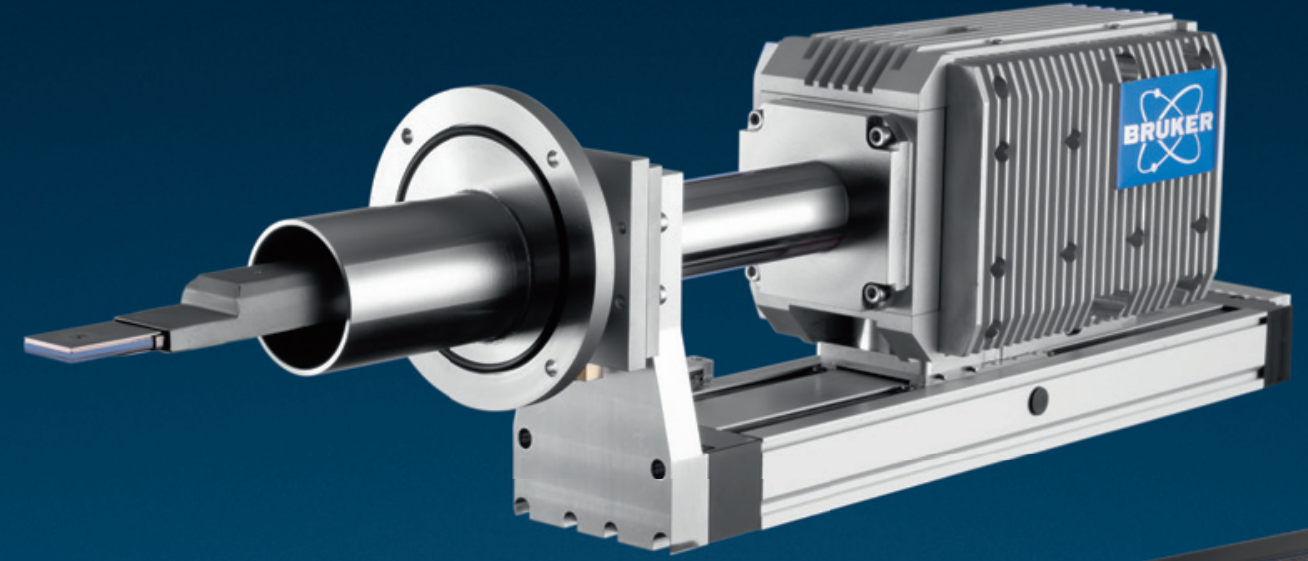

\section{Analyze textured samples with ease.}

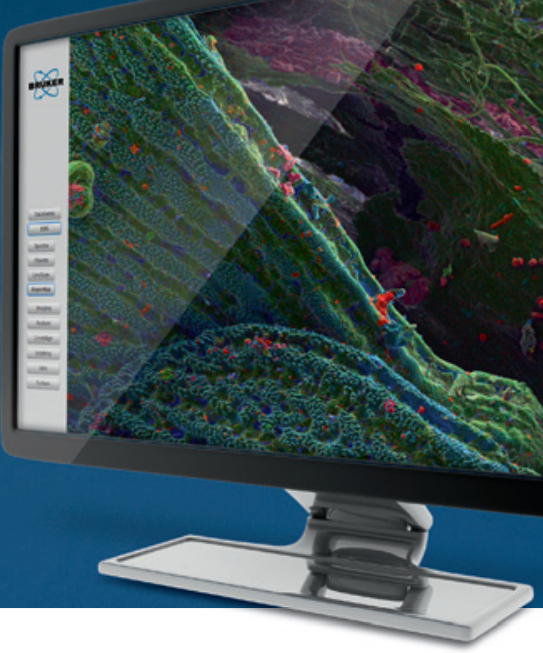

- The unique XFlash ${ }^{\circledR}$ FlatQUAD offers a large solid angle and an amazing take-off angle. This minimizes shadowing so you can see every nook and cranny of your sample in record time.

- Bruker's multiple detector systems provide large solid angles and great take-off angles too. You can start with one detector building up to four, depending on your needs.

- The Variable Z (VZ) adapter allows you to optimize take-off angles in-situ, which significantly improves the analysis of topographically challenging samples.

Someone has to be first.

\section{口t? www.bruker.com/quantax-flatquad is 国数}

